\title{
SCN5A allelic expression imbalance in African-Americans heterozygous for the common variant p.Ser1103Tyr
}

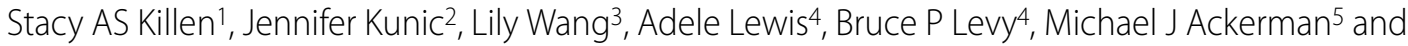 \\ Alfred L George Jr*2
}

\begin{abstract}
Background: Heterozygous and homozygous carriers of SCN5A-p.Ser1103Tyr, a common genetic variant with functional effects among African-Americans, have an increased risk of sudden death. We hypothesized that some heterozygous carriers may have unequal expression of wild-type and variant alleles and secondarily that predominance of the variant gene copy could further increase risk for sudden death in this population.

Methods: We quantified allele-specific expression of SCN5A-p.Ser1103Tyr by real-time reverse-transcription polymerase chain reaction (RT-PCR) in heart tissue from heterozygous African-American infants, who died from sudden infant death syndrome (SIDS) or from other causes, to test for allelic expression imbalance.

Results: We observed significant allelic expression imbalance in 13 of 26 (50\%) African-American infant hearts heterozygous for SCN5A-p.Ser1103Tyr, and a significant $(p<0.0001)$ bimodal distribution of $\log _{2}$ allelic expression ratios. However, there were no significant differences in the mean $\log _{2}$ allelic expression ratios in hearts of infants dying from SIDS as compared to infants dying from other causes and no significant difference in the proportion of cases with greater expression of the variant allele.
\end{abstract}

Conclusions: Our data provide evidence that SCN5A allelic expression imbalance occurs in African-Americans heterozygous for p.Ser1103Tyr, but this phenomenon alone does not appear to be a marker for risk of SIDS.

\section{Background}

Every year in the United States more than 300,000 people die suddenly from an unexpected cardiac event [1], with coronary artery disease accounting for $80 \%$ of sudden cardiac death (SCD) in the adult population [2]. However, in children and adolescents, inherited arrhythmias, such as long-QT syndrome, Brugada syndrome, familial atrial fibrillation, catecholaminergic polymorphic ventricular tachycardia, and Wolff-Parkinson-White syndrome, are a leading cause of sudden death [2]. Sudden death from cardiac causes is more common in African-Americans than in Caucasians [3,4]. In adults, sudden death from nonatherosclerotic heart disease is more frequent in African-Americans, while during infancy, African-Americans

\footnotetext{
* Correspondence: al.george@vanderbilt.edu

2 Department of Medicine, Vanderbilt University, Nashville, TN, USA Full list of author information is available at the end of the article
}

are twice as likely as Caucasians to die from sudden infant death syndrome (SIDS) [5].

Mutations in $S C N 5 A$, the gene encoding the poreforming $\alpha$-subunit of the cardiac voltage-gated sodium channel, have been associated with sudden death in long QT syndrome and Brugada syndrome [6]. A common SCN5A genetic variant (p.Ser1103Tyr) that is found in $13 \%$ of African-Americans is associated with an increased risk for cardiac arrhythmia and sudden cardiac death in that population $[7,8]$. Further, the p.Ser1103Tyr variant is over-represented in African-American SIDS victims as compared to non-SIDS infant death cases $[9,10]$ and sodium channels with the variant allele exhibit functional defects that may increase risk for life-threatening cardiac arrhythmias [7]. Because infants dying suddenly and unexpectedly are usually brought to autopsy, they provide a unique opportunity for studying tissue-specific expression of a potentially lethal genetic variant. 
Allelic variation in gene expression is a well-recognized phenomenon [11,12]. We hypothesized that AfricanAmericans heterozygous for SCN5A-p.Ser1103Tyr exhibit unequal expression of wild-type and variant alleles (allelic expression imbalance) and that predominance of the variant gene copy might further increase the risk for sudden death in this population.

\section{Methods}

\section{Tissue samples}

De-identified, frozen, postmortem heart tissues from African-American SIDS cases and African-American infant controls were obtained from two sources: the Tennessee Medical Examiner (29 cases, 16 controls), and a previously reported cohort collected at Mayo Clinic (10 cases, 30 controls) [10]. The SIDS cases from the Tennessee Medical Examiner met a rigorous definition of SIDS [13] or sudden unexpected infant death (SUID) [14]. Use of anonymous human tissues in this study was approved by the Institutional Review Board of Vanderbilt University.

\section{RNA extraction, cDNA synthesis, and genotyping}

Total RNA was extracted from pulverized heart muscle using TRIzol reagent (Invitrogen, Carlsbad, CA) according to the reagent supplier's instructions, followed by DNase digestion and final purification with RNeasy MinElute (Qiagen, Valencia, CA). RNA quality was determined by spectrophotometry and gel electrophoresis, and samples exhibiting degradation were not used. RNA samples were diluted to a concentration of $1 \mu \mathrm{g} / \mu \mathrm{l}$ prior to performing reverse transcription (RT) to synthesize cDNA used for genotyping and for allele-specific mRNA measurements. Reverse transcription was performed using $3 \mu \mathrm{g}$ RNA, $3.75 \mu \mathrm{M}$ random hexamers (Applied Biosystems, Foster City, CA), $0.5 \mathrm{mM}$ deoxynucleoside triphosphates (Roche, Basel, Switzerland), 1× M-MLV buffer (Promega, Madison, WI), 10 mM DTT, 40 units RNase inhibitor (Promega), and 200 units M-MLV reverse transcriptase (Promega). Reverse-transcription reactions were incubated sequentially for 10 minutes at $25^{\circ} \mathrm{C}, 50$ minutes at $42^{\circ} \mathrm{C}$, and 15 minutes at $70^{\circ} \mathrm{C}$. Genotyping for the $S C N 5 A$-p.Ser1103Tyr (c.3308C >A) variant was performed by direct sequencing of a $230 \mathrm{bp}$ amplicon (nucleotides 3217-3446 of the open reading frame of Genbank: NM 198056) generated by RT-PCR (primers: forward, TCCAGCAAGCAGCAGGAATC; reverse, TCAGCGGTGTTGGTCATGTC) from each RNA sample. The SCN5A-p.Ser1103Tyr variant has also been designated as p.Ser1102Tyr based on the amino acid sequence of an alternatively spliced transcript (Genbank: NM 000335). To be consistent with the numbering scheme used widely for SCN5A mutations, we used p.Ser1103Tyr based upon the canonical transcript.

\section{Allele-specific quantitative RT-PCR}

Heterozygous samples were evaluated for SCN5A allelic expression imbalance using real-time quantitative RTPCR and allele-specific TaqMan probes that contained a minor groove binder (MGB) at the 3 ' end (p.Ser1103: FAM-ACTGCCTCCTCTGA-MGB; p.Tyr1103: FAMACTGCCTACTCTGA-MGB; allele-specific sequences are underlined) to quantify the separate alleles. Amplification primers (forward: CCCTCCGGATTCCAGGAC; reverse: CTGCCCTCGGAGCAACTG) used in the assay generated a 156 bp amplicon (nucleotides 3264-3419 based on the open reading frame of Genbank: NM_198056) with a two-temperature cycling protocol $\left(95^{\circ} \mathrm{C}\right.$ for $20 \mathrm{~s}$ followed by 45 cycles of $95^{\circ} \mathrm{C}, 1 \mathrm{~s}$ and $60^{\circ} \mathrm{C}$, $20 \mathrm{~s})$. Under these conditions neither TaqMan probe exhibited cross-reactivity with the opposite allele. Amplicons corresponding to each allele were cloned into a plasmid vector and used as cDNA standards. All reactions were performed in triplicate on a 7900HT Fast Real-Time PCR system (Applied Biosystems). Individual reactions $(20 \mu \mathrm{l})$ contained $1 \times$ Fast Universal PCR Master Mix (Applied Biosystems), forward and reverse primers (3 $\mu \mathrm{M})$, an allele-specific probe $(10 \mu \mathrm{M})$, and $5 \mu \mathrm{l}$ of cDNA (or $5 \mu \mathrm{l}$ water for template-negative controls). The ability of the assay to quantitatively distinguish different proportions of the two alleles with high specificity was assessed using plasmid cDNA standards in the following mass ratios (p.Tyr1103:p.Ser1103): 4:1, 2:1, 1:1, 1:2, and 1:4. The cDNA standards were diluted to a final concentration of $0.001 \mathrm{ng} / \mu \mathrm{l}$ after preliminary studies determined that this concentration produced cycle threshold values similar to heart tissue RNA. Assays were performed in triplicate and each RNA sample was assayed from 2-3 independent preparations of cDNA.

\section{Data analysis}

Initial data analysis was performed using Applied Biosystems Sequence Detection System software (version 2.2.2). Two principles guided our approach to deduce allelic expression ratios. First, the cycle threshold $\left(\mathrm{C}_{\mathrm{T}}\right)$ is inversely proportional to the copy number of the template, assuming $100 \%$ amplification efficiency, and second, there is a linear relationship between $\log _{2}$ of the allele ratio and $\Delta C_{T}$ (difference between $C_{T}$ values for each allele). A standard curve generated from assaying cDNA standards mixed at known ratios and fitted with the equation $\log _{2}$ (p.Tyr1103:p.Ser1103) $=\alpha \Delta C_{T}+\beta(\alpha$ and $\beta$ are fit parameters) was used to interpolate the ratio of allele expression for case and control samples.

Statistical analysis was conducted using SAS (version 9.1.3, SAS institute, Cary, NC). Because multiple RT-PCR allelic imbalance assays were performed for each case and control, it was important to account for dependencies from repeated observations from the same subject. To 
this end, we used a mixed effects model to assess the allele ratios, with $\log _{2}$ (p.Tyr1103:p.Ser1103) as the outcome variable, with fixed effects, including sample source (Tennessee ME versus Mayo Clinic samples), age, gender, group (cases vs. controls), and random effects, including batch, subject and assay plate. The random batch effects were created based on unique combinations of PCR date and assay date. To obtain the estimates of Best Linear Unbiased Prediction (BLUP) of allelic expression imbalance for each subject, we constructed linear combinations of estimated parameters from this mixed effect model for each subject. Allelic imbalance was defined by $\log _{2}$ (p.Tyr1103:p.Ser1103) ratio significantly different from zero. The null hypothesis, $\log _{2}$ (p.Tyr1103:p.Ser1103) $=0$, describes the absence of allelic imbalance. A Bonferroni-corrected $\mathrm{p}$-value of $\mathrm{p}<$ 0.002 was considered statistically significant. We also plotted the p.Tyr1103:p.Ser1103 $\log _{2}$ allelic expression ratios for all cases and controls as a function of the count and used the Gaussian Fit option in OriginPro7 (OriginLab Corp., Northhampton, MA) to estimate the smoothed density function of the mean $\log _{2}$ allelic expression ratios. We then compared the goodness of fit of a two component mixture model and a normal model to the data using a likelihood ratio test.

\section{Results}

We identified 17 African-American SIDS cases and 9 African-American non-SIDS infant death controls that were heterozygous for SCN5A-p.Ser1103Tyr and that had available frozen heart tissue from which we were able to isolate intact, high quality total RNA. We developed a quantitative assay to measure allele-specific expression in heart tissue from African-American infants heterozygous for SCN5A-p.Ser1103Tyr using allele-specific real-time reverse-transcription PCR (RT-PCR). Allelic imbalance assays performed on monoallelic samples demonstrated negligible allele cross-reactivity.

Analysis of all subjects revealed that 13 of 26 (50\%) African-American heterozygous infant hearts exhibited $\log _{2}$ allelic expression ratios significantly different from zero ( $\mathrm{p}<0.002)$, indicating the presence of allelic expression imbalance. Further, we observed a significant $(\mathrm{p}<$ 0.0001 ) bimodal distribution of $\log _{2}$ allelic expression ratios (Fig. 1A). These data indicate that a subset of African-American infants who are heterozygous for p.Ser1103Tyr have unequal cardiac expression of the common and variant alleles. In individuals subjects, allelic expression imbalance was present in 10 of 17 cases (59\%) and 3 of 9 controls (33\%; Fig. 1B). However, analysis of allelic expression data using a mixed effects model demonstrated that the $\log _{2}$ p.Tyr1103:p.Ser1103 expression ratios for SIDS cases was not significantly different from that of the controls. Further, the proportion of cases with elevated variant allele expression was not statistically different from controls (Fisher's exact test, $\mathrm{p}>0.8$ ). Therefore, although our data provide significant evidence of $S C N 5 \mathrm{~A}$ allelic expression imbalance in heterozygous African-American hearts, there is no evidence for an association with the risk of SIDS.

\section{Discussion}

Inherited arrhythmias are a leading cause of sudden cardiac death in infants, children, and adolescents [2]. African-American infants have a two-fold higher risk of sudden death as compared with Caucasian infants [5]. One potential explanation of sudden death risk in African-Americans comes from work on the common genetic variant SCN5A-p.Ser1103Tyr. Thirteen percent of African-Americans carry at least one copy of the SCNSAp.Tyr1103 missense variant, for which in vitro evidence supports having a potential pathophysiologic role in increased cardiac arrhythmia risk [7]. Plant and colleagues demonstrated an over-representation of homozygous SCN5A-p.Tyr1103 subjects in a cohort of 133 African-American SIDS victims [9]. This study further demonstrated that SCN5A-p.Tyr1103 channels exhibit increased persistent sodium current (an arrhythmia susceptibility mechanism observed in LQTS) particularly when exposed to intracellular acidosis. A subsequent study demonstrated a significantly higher prevalence of heterozygous SCN5A-p.Ser1103Tyr carriers among 71 African-American cases of sudden unexplained infant deaths as compared with African-American controls [10].

We hypothesized that some African-Americans heterozygous for SCN5A-p.Ser1103Tyr have allelic expression imbalance with predominance of the variant gene copy. In this situation, greater relative expression of the minor allele (p.Tyr1103) might contribute more to arrhythmia susceptibility and further increase the risk of sudden cardiac death. Our data suggest that allelic expression imbalance occurs with $S C N 5 A$ but we did not observe a significant difference in allelic expression ratios or the proportion of cases with elevated variant expression. One potential limitation of our study stems from alternative splicing of $S C N 5 \mathrm{~A}$ mRNA. Our assay conditions do not distinguish expression of the two alleles present on differentially spliced SCN5A transcripts and therefore we can not exclude that allelic expression imbalance involves a specific splice variant.

Many autosomal genes can exhibit multi-fold differences in allele-specific expression even in healthy tissues $[11,12]$. In some settings, such as breast cancer associated with $B R C A 1$ or $B R C A 2$ mutations, allelic expression imbalance may underlie increased disease risk [15]. The most likely explanation for this phenomenon is the presence of cis-regulatory polymorphisms [16]. A prior study 


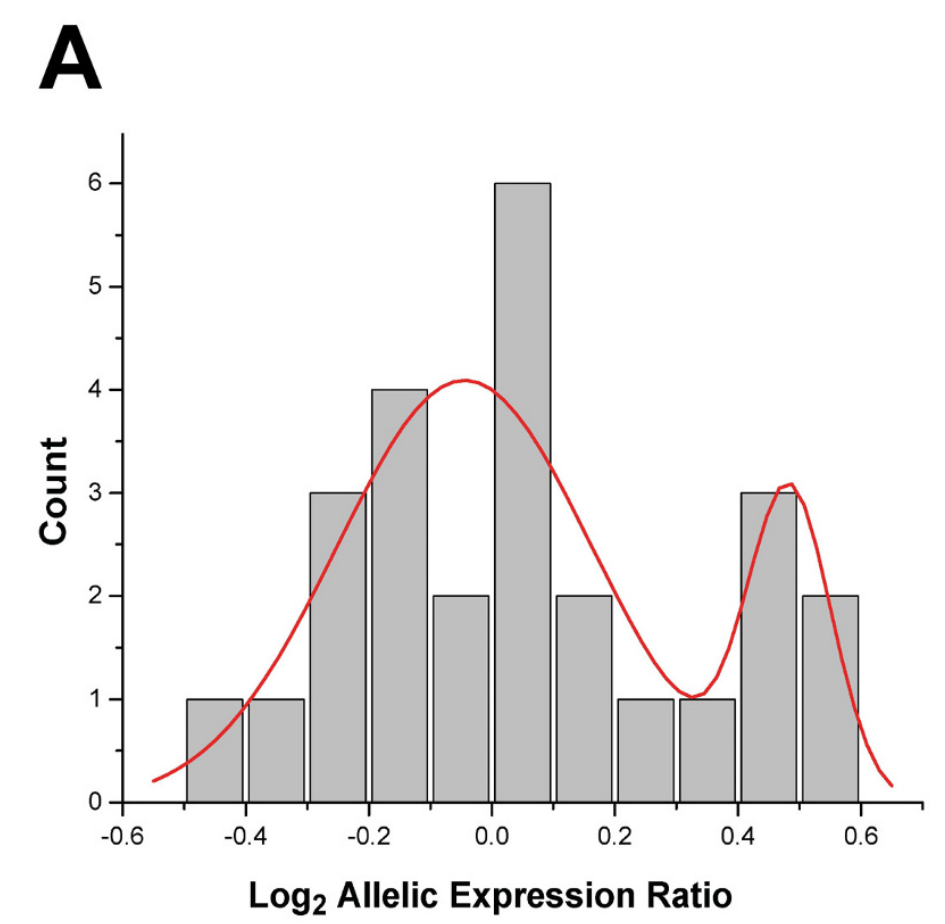

\section{B}

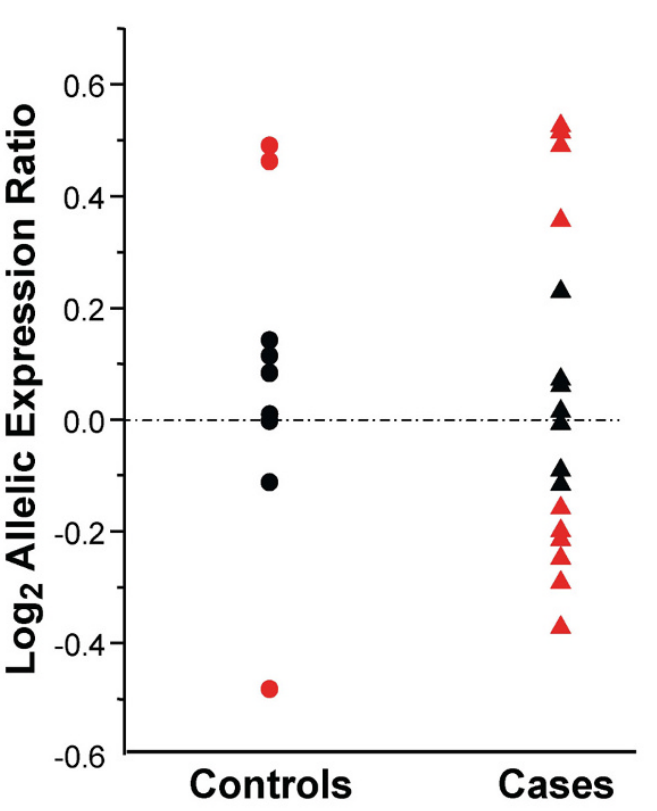

Figure 1 Allelic variation in cardiac SCN5A expression in African-American infants. (A) The $\log _{2}$ p.Tyr1103:p.Ser1103 allelic expression ratios for all cases and controls are plotted as a function of the count and fitted with Gaussian functions (red line). The goodness of fit for a two component model was significantly better than a single mode ( $<0.0001$ ), thus demonstrating a bimodal distribution of allelic expression ratios. (B) Distribution of $\log _{2}$ allelic expression ratios for SIDS cases and controls. Red colored symbols represent subjects with $\log _{2}$ allelic expression ratios significantly different from zero using a Bonferroni corrected level of significance $(p<0.002)$.

identified a common $S C N 5 A$ haplotype in Asians that was associated with significantly reduced promoter activity measured in vitro and correlated with variability in PR interval, QRS duration, and QRS widening during exposure to sodium channel blocking drugs [17]. This particular variant haplotype was not present in AfricanAmericans, and the study did not examine allelic variation in cardiac SCN5A expression. But this work did establish a precedent for predicting that allelic expression imbalance in SCN5A may occur and may have potential clinical consequences.

\section{Conclusions}

In conclusion, our study demonstrated that allelic variation in cardiac SCN5A expression occurs in AfricanAmericans heterozygous for p.Ser1103Tyr. This finding is biologically important because it demonstrates that SCN5A gene expression can exhibit allelic imbalance. However, this phenomenon alone was not associated with risk for SIDS in the cohorts we examined. Allelic expression imbalance in $S C N 5 A$ could be one mechanism among a host of genetic, epigenetic, and environmental phenomena that together modify sudden death risk in other susceptible hosts.

\section{Competing interests}

The authors declare that they have no competing interests.

\section{Authors' contributions}

SASK designed and performed experiments, analyzed data and wrote the manuscript. JK assisted with experiments and data analysis. LW contributed to statistical analysis of data. AL, BL and MJA procured tissue samples. ALG conceived of the study, helped plan experiments, analyzed data and wrote the manuscript. All authors have read and approved the final manuscript.

\section{Acknowledgements}

This work was supported by NIH grants HL083374 (A.L.G.) and HD42569 (M.J.A.) and institutional training grant T32-HL007256-30 (S.A.S.K.).

\section{Author Details}

'Department of Pediatrics, Vanderbilt University, Nashville, TN, USA, 2Department of Medicine, Vanderbilt University, Nashville, TN, USA,

${ }^{3}$ Department of Biostatistics, Vanderbilt University, Nashville, TN, USA, ${ }^{4}$ Tennessee Medical Examiner's Office, Nashville, TN, USA and ${ }^{5}$ Department of Pediatrics, Mayo School of Medicine, Rochester, MN, USA

Received: 26 October 2009 Accepted: 14 May 2010 Published: 14 May 2010

\section{References}

1. Arshad A, Mandava A, Kamath G, Musat D: Sudden cardiac death and the role of medical therapy. Prog Cardiovasc Dis 2008, 50:420-438.

2. Sarkozy A, Brugada P: Sudden cardiac death and inherited arrhythmia syndromes. J Cardiovasc Electrophysiol 2005, 16(Suppl 1):S8-S20.

3. Gillum RF: Sudden cardiac death in Hispanic Americans and African Americans. Am J Public Health 1997, 87:1461-1466. 
4. Burke AP, Farb A, Pestaner J, Malcom GT, Zieske A, Kutys R, Smialek J, Virmani R: Traditional risk factors and the incidence of sudden coronary death with and without coronary thrombosis in blacks. Circulation 2002, 105:419-424.

5. Pollack HA, Frohna JG: A competing risk model of sudden infant death syndrome incidence in two US birth cohorts. J Pediatr 2001, 138:661-667.

6. George AL Jr: Inheritable sodium channel diseases. In Cardiac Electrophysiology: From Cell to Bedside 5th edition. Edited by: Zipes DP, Jalife J. Philadelphia: Elsevier; 2009.

7. Splawski I, Timothy KW, Tateyama M, Clancy CE, Malhotra A, Beggs AH, Cappuccio FP, Sagnella GA, Kass RS, Keating MT: Variant of SCN5A sodium channel implicated in risk of cardiac arrhythmia. Science 2002, 297:1333-1336.

8. Burke A, Creighton W, Mont E, Li L, Hogan S, Kutys R, Fowler D, Virmani R: Role of SCN5A Y1102 polymorphism in sudden cardiac death in Blacks. Circulation 2005, 112:798-802.

9. Plant LD, Bowers PN, Liu Q, Morgan T, Zhang T, State MW, Chen W, Kittles RA, Goldstein SA: A common cardiac sodium channel variant associated with sudden infant death in African Americans, SCN5A S1103Y. J Clin Invest 2006, 116:430-435.

10. Van Norstrand DW, Tester DJ, Ackerman MJ: Overrepresentation of the proarrhythmic, sudden death predisposing sodium channel polymorphism $\mathrm{S} 1103 \mathrm{Y}$ in a population-based cohort of AfricanAmerican sudden infant death syndrome. Heart Rhythm 2008, 5:712-715.

11. Lo HS, Wang Z, Hu Y, Yang HH, Gere S, Buetow KH, Lee MP: Allelic variation in gene expression is common in the human genome. Genome Res 2003, 13:1855-1862.

12. Yan $H$, Yuan W, Velculescu VE, Vogelstein B, Kinzler KW: Allelic variation in human gene expression. Science 2002, 297:1143.

13. Krous HF, Beckwith JB, Byard RW, Rognum TO, Bajanowski T, Corey T, Cutz E, Hanzlick R, Keens TG, Mitchell EA: Sudden infant death syndrome and unclassified sudden infant deaths: a definitional and diagnostic approach. Pediatrics 2004, 114:234-238.

14. Corey TS, Hanzlick R, Howard J, Nelson C, Krous H: A functional approach to sudden unexplained infant deaths. Am J Forensic Med Pathol 2007, 28:271-277.

15. Chen X, Weaver J, Bove BA, Vanderveer LA, Weil SC, Miron A, Daly MB, Godwin AK: Allelic imbalance in BRCA1 and BRCA2 gene expression is associated with an increased breast cancer risk. Hum Mol Genet 2008, 17:1336-1348

16. Ge B, Pokholok DK, Kwan T, Grundberg E, Morcos L, Verlaan DJ, Le J, Koka V, Lam KC, Gagne V, et al:: Global patterns of cis variation in human cells revealed by high-density allelic expression analysis. Nature Genet 2009, 41:1216-1222.

17. Bezzina CR, Shimizu W, Yang P, Koopmann TT, Tanck MW, Miyamoto Y, Kamakura S, Roden DM, Wilde AA: Common sodium channel promoter haplotype in Asian subjects underlies variability in cardiac conduction. Circulation 2006, 113:338-344.

\section{Pre-publication history}

The pre-publication history for this paper can be accessed here: http://www.biomedcentral.com/1471-2350/11/74/prepub

\section{Submit your next manuscript to BioMed Central} and take full advantage of:

- Convenient online submission

- Thorough peer review

- No space constraints or color figure charges

- Immediate publication on acceptance

- Inclusion in PubMed, CAS, Scopus and Google Scholar

- Research which is freely available for redistribution

Submit your manuscript at www.biomedcentral.com/submit
C BioMed Central 